\title{
Tumor Identification Date
}

National Cancer Institute

\section{Source}

National Cancer Institute. Tumor Identification Date. NCI Thesaurus. Code C162178.

The date of tumor identification data collection. 